\title{
Systemic JIA genetically distinct
}

SJIA is
genetically
distinct from
other JIA
subtypes

Systemic juvenile idiopathic arthritis (sJIA) is phenotypically different from other forms of childhood-onset arthritis, set apart by systemic inflammatory features and a distinct response to anticytokine treatments. Now, the first genome-wide association study of juvenile idiopathic arthritis (JIA) reveals that sJIA is genetically distinct from other JIA subtypes. The findings suggest that the unique nature of sJIA is attributable, at least in part, to differences in its genetic basis.

JIA is an umbrella term referring to all forms of childhood arthritis that develop in the absence of a known cause, and the current classification scheme comprises seven mutually exclusive subtypes based on clinical and laboratory features. The new study found no evidence of shared genetic contribution between sJIA and oligoarticular JIA, rheumatoid factor (RF)-negative polyarticular JIA

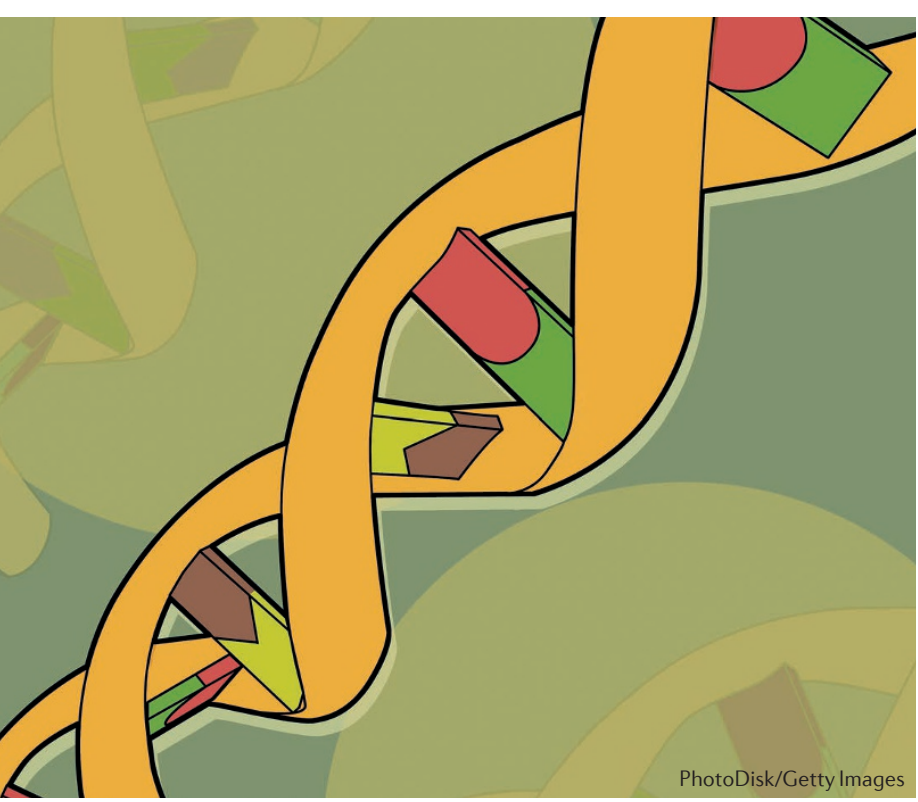

and RF-positive polyarticular JIA. "Based on our observations, we have called for sJIA to be recognized as a completely unique entity from any other form of childhood arthritis, that it should be removed from the JIA classification scheme and considered separately, and that the consideration of novel treatment modalities should be based on sJIA genetics and biology (instead of that of other forms of JIA)," argues Michael Ombrello, the study's corresponding author.

Ombrello and colleagues established the International Childhood Arthritis Genetics (INCHARGE) consortium - a collaboration of paediatric rheumatologists in nine countries across three continents to assemble the largest sJIA study population to date, which included 982 children with sJIA. "Genetic studies of sJIA to date have been candidate gene studies in small populations, usually from a single geographic site, and the findings have been modest associations at best," Ombrello explains. "The biggest obstacle to improving genetic studies of sJIA has been its rare nature, which has been the limiting factor in the assembly of larger case-control collections," he adds. Data from the cohort was paired with single nucleotide polymorphism (SNP) genotype data from geographically-matched healthy controls, giving a total study population of 8,992 .

The SNP datasets were stratified into nine geographically defined and ancestrally matched case-control collections. "Given the breadth of ancestral backgrounds, it was not possible to combine all of the populations into a single case-control collection without the detrimental influence of population stratification (leading to false positive results)," Ombrello explains. "Therefore, we decided to use a stratified approach, evaluating each of the nine case-control populations separately and then performing genome-wide meta-analysis to identify associations that transcended geography and ancestry."

Notably, the MHC locus was the strongest sJIA-risk locus identified in the study, confirming the association reported by the same group last year. A locus on the short arm of chromosome 1 (1p36.32) also showed an association with sJIA that exceeded the threshold for genome-wide significance. The study additionally identified 23 sJIA candidate risk loci, which had intermediate evidence of association, considered suggestive of a link with sJIA. "Importantly, none of these loci have been implicated in other forms of JIA or their adult-onset equivalents," points out Ombrello, "and none of the 'usual players' among autoimmune disease risk genes were within this group." Genetic risk scores and enrichment analysis demonstrated a lack of shared genetic architecture between sJIA and the most common forms of JIA.

Together, the findings of this first large-scale genomic study of sJIA support the notion that the pathophysiological mechanisms underlying sJIA are distinct from those of other subtypes of JIA, with potentially important implications for its classification as well as research into therapeutic interventions.

Sarah Onuora

ORIGINAL ARTICLE Ombrello, M. J. et al.

Genetic architecture distinguishes systemic juvenile idiopathic arthritis from other forms of juvenile idiopathic arthritis: clinical and therapeutic implications. Ann. Rheum. Dis. http://dx.doi.org/10.1136/annrheumdis2016-210324 (2016) 